\title{
Signature change from Schutz's canonical quantum cosmology and its classical analogue
}

\author{
Pouria Pedram, Shahram Jalalzadeh ${ }^{\dagger}$ \\ Department of Physics, Shahid Beheshti University, Evin, Tehran 19839, Iran
}

November 15, 2018

\begin{abstract}
We study the signature change in a perfect fluid Friedmann-Robertson-Walker quantum cosmological model. In this work the Schutz's variational formalism is applied to recover the notion of time. This gives rise to a Schrödinger-Wheeler-DeWitt equation with arbitrary ordering for the scale factor. We use the eigenfunctions in order to construct wave packets and evaluate the time-dependent expectation value of the scale factor which coincides with the ontological interpretation. We show that these solutions exhibit signature transitions from a finite Euclidean to a Lorentzian domain. Moreover, such models are equivalent to a classical system where, besides the perfect fluid, a repulsive fluid is present.
\end{abstract}

Pacs: 98.80.Qc, 04.40.Nr, 04.60.Ds

\section{Introduction}

The notion of signature transition mainly started to appear in the works of Hartle and Hawking [1, 2, 3, 4, where they argued that in quantum cosmology, amplitudes for gravity should be expressed as the sum of all compact Riemannian manifolds whose boundaries are located at the signature changing hypersurface. A signature changing spacetime is a manifold which contains both Euclidean and Lorentzian regions [5, 6]. In classical general relativity, the metric which represents signature change must be either degenerate (vanishing determinant) or discontinuous. On the other hand, Einstein's equations implicitly assume that the metric is non-degenerate and at least continuous [7].

In more recent times, a number of authors have studied this problem when a scalar field is coupled to Einstein's field equations and shown that the resulting solutions, when properly parameterized, exhibit signature transition [8, 9, 10, 11]. In a similar way, a classical model is studied in Ref. [12] in which a self-interacting scalar field is coupled to Einstein's equations with a Sinh-Gordon interaction potential. The field equations are

\footnotetext{
*Email: pouria.pedram@gmail.com

$\dagger$ Email: s-jalalzadeh@sbu.ac.ir
} 
solved exactly for the scale factor and scalar field which give rise to a spatially flat Friedmann-Robertson-Walker (FRW) cosmology with signature changing properties. The case of the non-flat Universe is addressed in 13 with a discussion about the conditions under which signature transition exists. It is well known that in classical signature change spacetimes, we have some junction conditions on the signature changing hypersurface. On the other hand, there is no any satisfactory unique junction condition (see [14, 15] and references therein). At the quantum cosmology level the same issue is investigated in Ref. [16] with an analysis pertaining to the exact solutions of the Wheeler-DeWitt equation. Signature transition has also been studied in multi-dimensional classical and quantum cosmology [17] where a $4+d$-dimensional spacetime is minimally coupled to a scalar field. The coupling between spinor field and gravity based on FRW and Bianchi cosmological models are also addressed in Refs. [18, 19, respectively. Also, it has been used as a compactification mechanism for KaluzaKlein cosmology [20, 21] with a cosmological constant. Finally, the issue of the classically signature change in the brane world models is studied in [22].

In this paper, we consider a smooth signature changing type spacetime. First, we study the classical solutions with one component perfect fluid and show that the solutions do not extend to the Euclidian region. Then, we construct the corresponding quantum cosmological model via Schutz's variational formalism which has been attracted much attentions in recent times [23, 24]. By canonical quantization of this model, we can avoid the singularity and consequently, we obtain the signature changing type metric. At last, we show that the quantum signature change scenario can be reproduced exactly by a classical model where a repulsive fluid is added to the normal perfect fluid. The repulsive fluid can be given by a stiff matter equation of state $p=\rho$, independently of the content of the normal fluid.

The paper is organized as follows: In Sec. 2, the quantum cosmological model with a perfect fluid as the matter content is constructed in Schutz's formalism [25, and the Schrödinger-Wheeler-DeWitt (SWD) equation in minisuperspace is written down to quantize the model. The wave function depends on the scale factor $a$ and the canonical variable associated to the fluid, which in the Schutz's variational formalism plays the role of time $T$. We separate the wave function into two parts, one depending solely on the scale factor and the other depending only on the time. The solution in the time sector of the SWD equation is trivial, leading to imaginary exponentials of type $e^{i E T}$, where $E$ is the energy of the system. Then, we construct the wave packets 
from the eigenfunctions and compute the time-dependent expectation value of the scale factors. We analyze the results in the context of signature change and construct the classical analogue of quantum solutions via adding a repulsive perfect fluid. In Sec. 3, we present our conclusions.

\section{The model}

The action for gravity plus perfect fluid in Schutz's formalism is written as

$$
\mathcal{A}=\int_{M} d^{4} x \sqrt{-g} R+2 \int_{\partial M} d^{3} x \sqrt{h} h_{a b} K^{a b}+\int_{M} d^{4} x \sqrt{-g} p
$$

where $h_{a b}$ is the induced metric over the three-dimensional spatial hypersurface, which is the boundary $\partial M$ of the four dimensional manifold $M$ and $K^{a b}$ is the extrinsic curvature. We choose units such that the factor $16 \pi G$ becomes equal to one. The first two terms were first obtained in [26] and the last term (1) represents the matter contribution to the total action. Perfect fluid satisfies the barotropic equation of state

$$
p=\alpha \rho
$$

In Schutz's formalism [25] the fluid's four-velocity is expressed in terms of five potentials $\epsilon, \zeta, \beta, \theta$ and $S$ :

$$
u_{\nu}=\frac{1}{\mu}\left(\epsilon_{, \nu}+\zeta \beta_{, \nu}+\theta S_{, \nu}\right)
$$

where $\mu$ is the specific enthalpy and $S$ is the specific entropy. The potentials $\zeta$ and $\beta$ are connected with rotation and are absent in FRW models and the variables $\epsilon$ and $\theta$ have no clear physical meaning. The four-velocity satisfies the following normalization condition

$$
u^{\nu} u_{\nu}=-1
$$

The Hartle-Hawking no-boundary proposal [1, 3, 4, implies that spacetime is partly Euclidian and partly Lorentzian. The motivation of this proposal involves the path integral formulation of quantum gravity. To have a feeling about the quantum theory it is necessary to have an understanding of the associated classical theory by constructing the classical spacetime with signature changing structure. In fact, there are two main proposals for this purpose. In the first proposal, the metric of spacetime is everywhere non-degenerate but fails to be continuous at the surface that divides the Euclidian from the Lorentzian region. On the other hand, 
in the second proposition, metric is everywhere smooth but is degenerate at the surface of signature change [8, 10, 27, 28.

Here, we are interested to use the second one. The authors of Ref. [29] have shown that for smooth signature changing spacetime there exist coordinates such that

$$
d s^{2}=-N^{2}(t) t d t^{2}+h_{i j} d x^{i} d x^{j}
$$

For this case, Kossowski and Kriele [30] have shown that the energy-momentum tensor of the matter field becomes bounded if and only if the signature change hypersurface $(\Sigma)$ is totally geodesic and $\partial_{t} h_{i j}=0$ at $\Sigma$.

To proceed further, let us consider the signature changing FRW metric as

$$
d s^{2}=-N^{2}(t) t d t^{2}+a^{2}(t) g_{i j} d x^{i} d x^{j}
$$

where $N^{2}>0$ and is now inserted in action (11). In this expression, $N(t)$ is the lapse function and $g_{i j}$ is the metric on the constant-curvature spatial section. Using the constraints for the fluid, and after some thermodynamical considerations and dropping the surface terms, the final reduced action takes the form [31].

$$
\mathcal{A}=\int d t\left[-6 \frac{\dot{a}^{2} a}{t^{1 / 2} N}+6 \kappa N t^{1 / 2} a+N^{-1 / \alpha} t^{-1 / 2 \alpha} a^{3} \frac{\alpha}{(\alpha+1)^{1 / \alpha+1}}(\dot{\epsilon}+\theta \dot{S})^{1 / \alpha+1} \exp \left(-\frac{S}{\alpha}\right)\right]
$$

where dot denotes the derivation with respect to $t$. The reduced action may be further simplified using canonical methods [3] resulting in the super-Hamiltonian

$$
\mathcal{H}=-\frac{p_{a}^{2}}{24 a}-6 \kappa a+\frac{p_{\epsilon}^{\alpha+1} e^{S}}{a^{3 \alpha}}
$$

where $p_{a}=-12 \dot{a} a / t^{1 / 2} N, p_{\epsilon}=\rho_{0} a^{3}$ and $\rho_{0}=\frac{\mu^{1 / \alpha}}{(1+\alpha)^{1 / \alpha}} e^{-S / \alpha}$ is the rest mass density of the fluid. The following additional canonical transformations

$$
\begin{aligned}
T=-p_{S} e^{-S} p_{\epsilon}^{-(\alpha+1)}, & p_{T}=p_{\epsilon}^{\alpha+1} e^{S}, \\
\bar{\epsilon}=\epsilon-(\alpha+1) \frac{p_{S}}{p_{\epsilon}}, & \bar{p}_{\epsilon}=p_{\epsilon},
\end{aligned}
$$

simplify the super-Hamiltonian to

$$
\mathcal{H}=-\frac{p_{a}^{2}}{24 a}-6 \kappa a+\frac{p_{T}}{a^{3 \alpha}}
$$


where the momentum $p_{T}$ is the only remaining canonical variable associated with matter. It appears linearly in the super-Hamiltonian. The parameter $\kappa$ defines the curvature of the spatial section, taking the values $0,1,-1$ for a flat, positive-curvature or negative-curvature Universe, respectively.

The classical dynamics is governed by the Hamilton equations, derived from Eq. (10) and Poisson brackets as

$$
\left\{\begin{array}{l}
\dot{a}=\quad\left\{a, t^{1 / 2} N \mathcal{H}\right\}=-\frac{t^{1 / 2} N p_{a}}{12 a}, \\
\dot{p_{a}}=\left\{p_{a}, t^{1 / 2} N \mathcal{H}\right\}=-\frac{t^{1 / 2} N p_{a}^{2}}{24 a^{2}}+6 t^{1 / 2} N \kappa+3 \alpha t^{1 / 2} N \frac{p_{T}}{a^{3 \alpha+1}} \\
\dot{T}=\left\{T, t^{1 / 2} N \mathcal{H}\right\}=t^{1 / 2} N a^{-3 \alpha} \\
\dot{p_{T}}=\left\{p_{T}, t^{1 / 2} N \mathcal{H}\right\}=0 .
\end{array}\right.
$$

Choosing the gauge $N=a^{3 \alpha}$, we have $T=\frac{2}{3} t^{\frac{3}{2}}$ and the following constraint equation $\mathcal{H}=0$

$$
-\frac{6 \dot{a}^{2}}{t a^{3 \alpha-1}}-6 \kappa a^{3 \alpha+1}+p_{T}=0
$$

For the flat case $(\kappa=0)$, we have the following solutions for $\alpha \neq 1$

$$
a(t)=\left\{\sqrt{\frac{p_{T}}{6}}(1-\alpha)\right\}^{\frac{2}{3(1-\alpha)}} t^{\frac{1}{1-\alpha}}
$$

and $\alpha=1$ (stiff matter)

$$
a(t)=a_{0} e^{\frac{2}{3} \sqrt{\frac{P_{T}}{6}} t^{3 / 2}} .
$$

Stiff matter is a fluid with pressure equal to the energy density and speed of sound equal to speed of light. Although at $t=0$ (signature changing hypersurface) this solution is finite and the energy-momentum tensor is bounded, for $t<0$ the scale factor becomes complex. Moreover, for $\alpha \neq 1$ the scale factor vanishes and the energy-momentum tensor is not bounded. Therefore, we can not construct the signature changing spacetime using simple perfect fluid in the classical domain. In fact, in order to have this type of spacetime in classical cosmology, we need some kinds of scalar fields with suitable potential term that can provide the signature change [12].

Imposing the standard quantization conditions on the canonical momenta and demanding that the superHamiltonian operator annihilate the wave function, we are led to the following SWD equation in the minisu- 
perspace with general factor ordering $(\hbar=1)$

$$
\frac{1}{2}\left(\frac{1}{a^{i}} \frac{\partial}{\partial a} \frac{1}{a^{j}} \frac{\partial}{\partial a} \frac{1}{a^{k}}+\frac{1}{a^{k}} \frac{\partial}{\partial a} \frac{1}{a^{j}} \frac{\partial}{\partial a} \frac{1}{a^{i}}\right) \psi(a, T)-144 \kappa a \psi(a, T)-i 24 a^{-3 \alpha} \frac{\partial \psi(a, T)}{\partial T}=0 .
$$

Where $i+j+k=1$. Equation (15) takes the form of a Schrödinger equation $i \partial \Psi / \partial T=\hat{H} \Psi$. This operator is formally self-adjoint for any choice of the ordering parameters $i, j, k$ with the standard inner product

$$
(\Phi, \Psi)=\int_{0}^{\infty} a^{-3 \alpha} \Phi^{*} \Psi d a
$$

Moreover, the wave functions should satisfy the restrictive boundary conditions of which the simplest ones are

$$
\Psi(0, T)=0 \quad \text { or }\left.\quad \frac{\partial \Psi(a, T)}{\partial a}\right|_{a=0}=0
$$

The SWD equation (15) can be solved by separation of variables as

$$
\psi(a, t)=e^{i E T} \psi(a)
$$

where the $a$ dependent part of the wave function $(\psi(a))$ satisfies

$$
\frac{1}{2}\left(\frac{1}{a^{i}} \frac{\partial}{\partial a} \frac{1}{a^{j}} \frac{\partial}{\partial a} \frac{1}{a^{k}}+\frac{1}{a^{k}} \frac{\partial}{\partial a} \frac{1}{a^{j}} \frac{\partial}{\partial a} \frac{1}{a^{i}}\right) \psi(a)-144 \kappa a \psi(a)+24 E a^{-3 \alpha} \psi(a)=0 .
$$

For flat case $(\kappa=0)$, this equation reduces to

$$
\left[\frac{1}{2}\left(\frac{1}{a^{i}} \frac{\partial}{\partial a} \frac{1}{a^{j}} \frac{\partial}{\partial a} \frac{1}{a^{k}}+\frac{1}{a^{k}} \frac{\partial}{\partial a} \frac{1}{a^{j}} \frac{\partial}{\partial a} \frac{1}{a^{i}}\right)+24 E a^{-3 \alpha}\right] \psi(a)=0 .
$$

Now, using the relation

$$
\left(\frac{1}{a^{i}} \frac{\partial}{\partial a} \frac{1}{a^{j}} \frac{\partial}{\partial a} \frac{1}{a^{k}}\right) \psi(a)=a^{-1} \frac{\partial^{2} \psi(a)}{\partial a^{2}}-(2 k+j) a^{-2} \frac{\partial \psi(a)}{\partial a}+k(k+j+1) a^{-3} \psi(a)
$$

we can rewrite the equation (20) as

$$
\psi^{\prime \prime}-a^{-1} \psi^{\prime}+\left[\frac{1}{2}(i(i+j+1)+k(k+j+1)) a^{-2}+24 E a^{-3 \alpha+1}\right] \psi=0,
$$

where a prime denotes the derivation with respect to $a$. Equation (22) admits a solution under the form of Bessel functions, leading to the following final expression for the stationary wave functions

$$
\Psi_{E}(a, T)=e^{i E T} a\left[c_{1} J_{l}\left(\frac{\sqrt{96 E}}{3(1-\alpha)} a^{\frac{3(1-\alpha)}{2}}\right)+c_{2} Y_{l}\left(\frac{\sqrt{96 E}}{3(1-\alpha)} a^{\frac{3(1-\alpha)}{2}}\right)\right],
$$


where $l=\frac{2 \sqrt{1-\frac{1}{2}(i(i+j+1)+k(k+j+1))}}{3(1-\alpha)}$. Now, the wave packets can be constructed by superposing these eigenfunctions with the following structure

$$
\Psi(a, T)=\int_{0}^{\infty} A(E) \Psi_{E}(a, T) d E .
$$

We choose $c_{2}=0$, for satisfying the first boundary condition (17). By choosing $A(E)$ as a quasi-gaussian weight factor and defining $r=\frac{\sqrt{96 E}}{3(1-\alpha)}$, an analytical expression for the wave packet can be found

$$
\Psi(a, T)=a \int_{0}^{\infty} r^{l+1} e^{-\gamma r^{2}+i \frac{3}{32}(1-\alpha)^{2} r^{2} T} J_{l}\left(r a^{\frac{3(1-\alpha)}{2}}\right) d r
$$

where $\gamma$ is an arbitrary positive constant. The above integral is known [32, and the wave packet takes the form

$$
\Psi(a, T)=a^{\frac{3}{2} l(1-\alpha)+1}(2 B)^{-l-1} e^{-\frac{a^{3(1-\alpha)}}{4 B}},
$$

where $B=\gamma-i \frac{3}{32}(1-\alpha)^{2} T$. Following the many worlds interpretation of quantum mechanics [33], we may write the expectation value for the scale factor $a$ as

$$
\langle a\rangle(T)=\frac{\int_{0}^{\infty} a^{1-3 \alpha} \Psi(a, T)^{*} \Psi(a, T) d a}{\int_{0}^{\infty} a^{-3 \alpha} \Psi(a, T)^{*} \Psi(a, T) d a} .
$$

which yields

$$
\langle a\rangle(t)=\frac{\Gamma\left(\frac{l}{2}+1\right)}{\Gamma\left(\frac{l}{2}+\frac{2-3 \alpha}{3-3 \alpha}\right)}\left[\frac{2}{\gamma}\left(\frac{1}{256}(1-\alpha)^{4} t^{3}+\gamma^{2}\right)\right]^{\frac{1}{3(1-\alpha)}} .
$$

where we have used $T=\frac{2}{3} t^{\frac{3}{2}}$. These solutions, asymptotically correspond to the flat classical models for the late times

$$
a(t) \propto t^{1 /(1-\alpha)} .
$$

We can also study the situation from the ontological interpretation of quantum mechanics 34, 35. In this approach the wave function can be written as

$$
\Psi(a, T)=R e^{i S},
$$

where $R$ and $S$ are real functions. Inserting this expression in the SWD equation (15), for $\kappa=0$ we have

$$
\begin{aligned}
\frac{1}{a^{3 \alpha}} \frac{\partial S}{\partial T}-\frac{1}{24 a}\left(\frac{\partial S}{\partial a}\right)^{2}+Q & =0, \\
\frac{\partial R}{\partial T}-\frac{1}{12 a^{1-3 \alpha}}\left(\frac{\partial R}{\partial a} \frac{\partial S}{\partial a}+R \frac{\partial^{2} S}{\partial a^{2}}-\frac{1}{a} R \frac{\partial S}{\partial a}\right) & =0,
\end{aligned}
$$


where

$$
Q=\frac{1}{24 a} \frac{1}{R}\left(\frac{\partial^{2} R}{\partial a^{2}}-\frac{1}{a} \frac{\partial R}{\partial a}\right)+\frac{i(i+j+1)+k(k+j+1)}{48 a^{3}}
$$

is the quantum potential which modifies the Hamilton-Jacobi equation (31) and the last term is related to factor ordering. When the quantum potential is more important than the classical potential, we can expect a behavior deviating from the classical one. Note that in the present case, since $\kappa=0$, the classical potential is zero. The wave function (26) implies

$$
\begin{aligned}
R & =a^{\frac{3}{2} l(1-\alpha)+1}\left[4 \gamma^{2}+\left(\frac{3}{16}\right)^{2}(1-\alpha)^{4} T^{2}\right]^{-(l+1) / 2} \exp \left\{-\frac{\gamma a^{3(1-\alpha)}}{4\left[\gamma^{2}+\left(\frac{3}{32}\right)^{2}(1-\alpha)^{4} T^{2}\right]}\right\} \\
S & =-\frac{3}{128} \frac{(1-\alpha)^{2} a^{3(1-\alpha)} T}{\left[\gamma^{2}+\left(\frac{3}{32}\right)^{2}(1-\alpha)^{4} T^{2}\right]}-(l+1) \arctan \left[\frac{3}{32} \frac{(1-\alpha)^{2} T}{\gamma}\right] .
\end{aligned}
$$

The Bohmian trajectories, which determine the behavior of the scale factor, are given by

$$
p_{a}=\frac{\partial S}{\partial a}
$$

Using the above definition, the equation for the Bohmian trajectories becomes

$$
\frac{512}{a} \frac{d a}{d T}=3(1-\alpha)^{3} \frac{T}{\left[\gamma^{2}+\left(\frac{3}{32}\right)^{2}(1-\alpha)^{4} T^{2}\right]}
$$

which can be integrated to

$$
a(T)=a_{0}\left[\gamma^{2}+\left(\frac{3}{32}\right)^{2}(1-\alpha)^{4} T^{2}\right]^{\frac{1}{3(1-\alpha)}}
$$

where $a_{0}$ is an integration constant. This result coincides with the one which is found by computation of the expectation value of the scale factor (28). The quantum potential takes the form

$$
\begin{array}{r}
Q(a, T)=\frac{3}{32 a^{3 \alpha}} \frac{\gamma(1-\alpha)^{2}}{\left[\gamma^{2}+\left(\frac{3}{32}\right)^{2}(1-\alpha)^{4} T^{2}\right]}\left\{\frac{\gamma a^{3(1-\alpha)}}{\left[\gamma^{2}+\left(\frac{3}{32}\right)^{2}(1-\alpha)^{4} T^{2}\right]}-(l+1)\right\} \\
+\frac{2\left(\frac{9}{4} l^{2}(1-\alpha)^{2}-1\right)+i(i+j+1)+k(k+j+1)}{48 a^{3}} .
\end{array}
$$

Now, using the solution (38), we can find the the quantum potential in terms of the scale factor as

$$
\begin{aligned}
Q(a) & =-\frac{1}{48 a^{3}}\left[\frac{9}{2} \gamma a_{0}^{3(1-\alpha)}(\alpha-1)\left(\gamma a_{0}^{3(1-\alpha)}-(l+1)\right)-2\left(\frac{9}{4} l^{2}(1-\alpha)^{2}-1\right)-i(i+j+1)-k(k+j+1)\right] \\
& :=-\frac{C}{a^{3}} .
\end{aligned}
$$


It is clear that the quantum effects become important near $a=0$ and become negligible for large values of the scale factor. The avoidance of the singularity is due to the repulsive force $F_{a}=-\partial Q(a, T) / \partial a$ extracted from the quantum potential. To show this, we can write the super-Hamiltonian for the flat case in Bohmian picture as

$$
\mathcal{H}_{B}=-\frac{6 a \dot{a}^{2}}{N^{2} t}+\frac{p_{T}}{a^{3 \alpha}}-\frac{C}{a^{3}} .
$$

Now, the zero energy condition yields

$$
\frac{6}{N^{2} t}\left(\frac{\dot{a}}{a}\right)^{2}=\frac{p_{T}}{a^{3(\alpha+1)}}-\frac{C}{a^{6}}
$$

The sign of the left hand side of the above equation is negative for the negative values of $t$ and positive for $t>0$. Consequently the sign of the right hand side changes as well. Hence the right hand side vanishes at $t=0$. Now, since we have solutions both for $t<0$ and $t>0$, there should therefore exist signature changing hypersurface so that $a \propto\left(C / P_{T}\right)^{1 / 3(1-\alpha)}=a_{0}$. Also it is easy to see from equation (42) that the scale factor is less than $a_{0}$ for negative values of $t$ and grater than $a_{0}$ for the positive values of $t$. Hence, this equation predicts the existence of three regions, namely, a Lorentzian domain, a signature changing hypersurface and an Euclidean domain. Therefore, since the discussion above is independent of the choice of $N$, the quantum signature change behavior is in fact gauge independent.

The solutions (28) show a continuous transition from a finite Euclidean domain to the Lorentzian one. It is easy to show that

$$
\begin{aligned}
\left.\partial_{t}\langle a\rangle\right|_{t=0} & =0, \\
\left.\partial_{t} \partial_{t}\langle a\rangle\right|_{t=0} & =0,
\end{aligned}
$$

which satisfy the Kossowski and Kriele mentioned theorem. Hence, in general, the quantum model predicts a signature change model when the singularity is approached. Moreover, the quantum effect leads to a repulsive force which results in a regular transition from Euclidean to the Lorentzian region.

The above discussion shows that one of the curious features of quantum cosmology is the use of Riemannian signature spaces to explain the origin of the observable Lorentzian signature Universe. There are various interpretations of this, the simplest of which is that the signature of the universe was initially Riemannian and 
then subsequently changed. It may be argued that the Lorentzian signature is an independent assumption of relativity rather than a consequence, with the theory being equally valid for Riemannian signature, and that in a quantum theory of gravity it would be unnatural to impose signature restrictions on the metric. The question arises as to whether the qualitative predictions of quantum cosmology can be obtained from purely classical relativity by relaxing the assumption of Lorentzian signature. Also in order to understand the quantum theory it is necessary to have an understanding of the associated classical theory i.e., the theory of classical spacetimes with signature type change.

Now, we try to construct the classical analogous to the quantum signature change cosmology. One way of implementing a repulsive phase in classical cosmology is to consider two fluids, one that acts attractively, and the other that acts repulsively [36. It is desirable that the repulsive fluid dominates for the small values of the scale factor, whereas the attractive fluid dominates for the large values of the scale factor. For the flat case, we can obtain the possible model in signature change coordinate (마) as

$$
\frac{1}{N^{2} t}\left(\frac{\dot{a}}{a}\right)^{2}=8 \pi G\left(\rho_{M}-\rho_{Q}\right)=\frac{C_{1}}{a^{m}}-\frac{C_{2}}{a^{n}}
$$

where $p_{M}=\alpha_{M} \rho_{M}, p_{Q}=\alpha_{Q} \rho_{Q}, m=3\left(1+\alpha_{M}\right)$ and $n=3\left(1+\alpha_{Q}\right)$. The subscripts $M$ and $Q$ stand for "normal" matter component and for "quantum" repulsive component, respectively.

Since the normal matter corresponds to $\alpha_{M}=-1,0, \frac{1}{3}$, and it is also desirable that the repulsive component dominates at small values of the scale factor, we choose $\alpha_{Q}>\frac{1}{3}$. With due attention to (42), we choose a repulsive stiff matter fluid $\alpha_{Q}=1$, which leads to $n=6$. Then, the solution is

$$
\frac{1}{N^{2} t}\left(\frac{\dot{a}}{a}\right)^{2}=\frac{C_{1}}{a^{3(1+\alpha)}}-\frac{C_{2}}{a^{6}}
$$

This equation can be solved by reparametrizing the time coordinate as

$$
t^{1 / 2} d t=d T
$$

which results in

$$
\left(\frac{a^{\prime}}{a}\right)^{2}=C_{1} a^{-3(1-\alpha)}-C_{2} a^{-6(1-\alpha)}
$$

where the prime means derivation with respect to $T$. This equation can be easily solved, leading to the following 
expression for the scale factor

$$
a(T)=\left(\frac{C_{1}}{C_{2}}\right)^{\frac{1}{3(1-\alpha)}}\left[\frac{C_{1}^{2} C_{2}}{36(1-\alpha)^{2}} T^{2}+1\right]^{\frac{1}{3(1-\alpha)}},
$$

which coincides with the quantum mechanical solution with only the ordinary perfect fluid (28). Therefore, the quantum solutions are equivalent to the classical solutions where gravity is coupled to the same perfect fluid plus a repulsive fluid with a stiff matter equation of state $p_{Q}=\rho_{Q}$.

The comparison between classical (49) and quantum (28) solutions can fix $C_{1}$ and $C_{2}$ as

$$
C_{1}=\left(\frac{\Gamma\left(\frac{l}{2}+1\right)}{\Gamma\left(\frac{l}{2}+\frac{2-3 \alpha}{3-3 \alpha}\right)}\right)^{1-\alpha} \frac{3}{8} 3^{1 / 3} \frac{(1-\alpha)^{2}}{\gamma^{1 / 3}}, \quad C_{2}=\left(\frac{\Gamma\left(\frac{l}{2}+1\right)}{\Gamma\left(\frac{l}{2}+\frac{2-3 \alpha}{3-3 \alpha}\right)}\right)^{-2(1-\alpha)} \frac{3}{4} 3^{1 / 3} \frac{(1-\alpha)^{2}}{\gamma^{4 / 3}} .
$$

Now we can check the null energy condition $(\rho+p \geq 0)$ in order to investigate the avoidance of the singularity in comoving coordinate.

The existence of a repulsive term implies that the energy conditions are violated as the singularity is approached, leading to its avoidance. If we define $\rho_{\text {eff }}$ and $p_{\text {eff }}$ as the sum of the energy and pressure for both attractive and repulsive fluids and using the solutions (49), with an unimportant absorbtion of integration constant in the definition of the time coordinate, we have

$$
\begin{aligned}
\rho_{e f f} & +p_{e f f}=\frac{4}{a^{6 \alpha}}\left(-\frac{a^{\prime \prime}}{a}+(1+3 \alpha) \frac{a^{2}}{a^{2}}\right) \\
& =\frac{1}{a^{6 \alpha}} \frac{8}{3(1-\alpha)^{2}}\left[\frac{(1+\alpha) T^{2}-(1-\alpha)}{\left(T^{2}+1\right)^{2}}\right],
\end{aligned}
$$

which is negative for $T<\sqrt{\frac{1-\alpha}{1+\alpha}}$. Therefore, for $\alpha<1$ the null energy condition is violated around the signature change hypersurfaces.

Repulsive gravitational effects in classical general relativity can also be generated by self interacting scalar fields to which an effective energy density and an effective pressure can be associated such that

$$
\left\{\begin{array}{l}
p_{\phi}=\frac{\dot{\phi}^{2}}{2}-U(\phi), \\
\rho_{\phi}=\frac{\dot{\phi}^{2}}{2}+U(\phi) .
\end{array}\right.
$$

A convenient choice for the potential $U(\phi)$ may lead to the repulsive effect and consequently we have classical signature change. According to our discussions of need to specific kind of matter to have signature changing, we assume that the scalar field is dominated at early Universe and we choose a specific form of the potential 

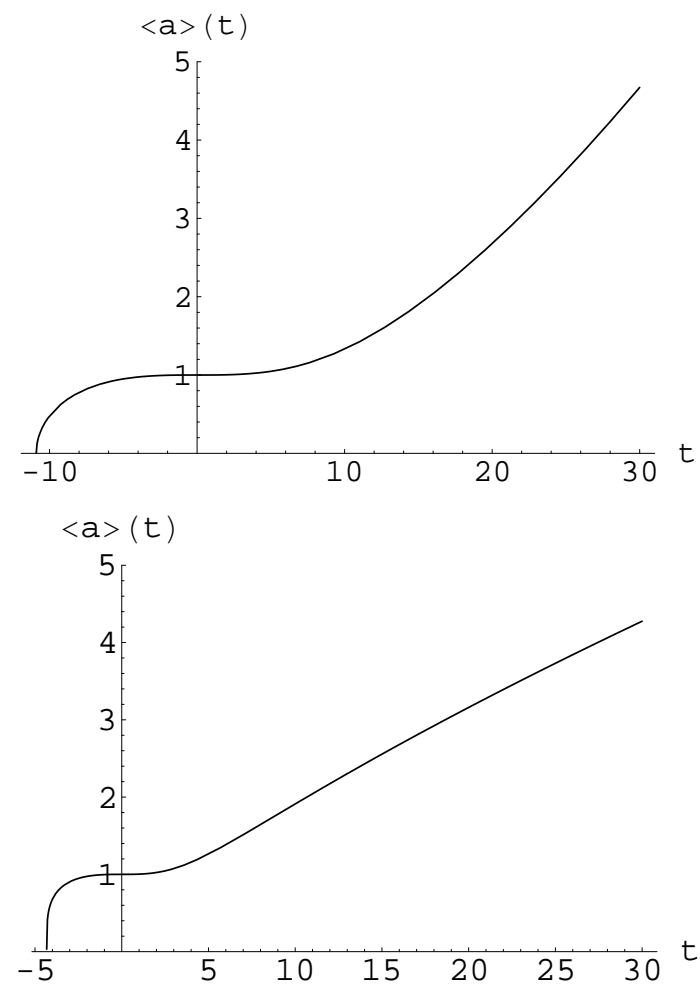

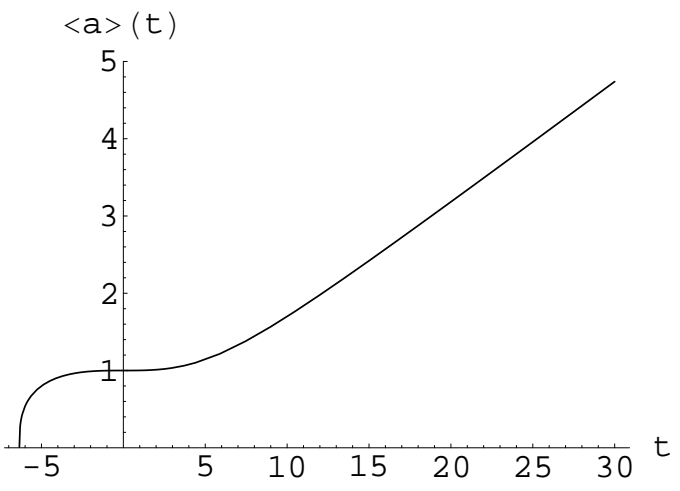

$<a>(t)$

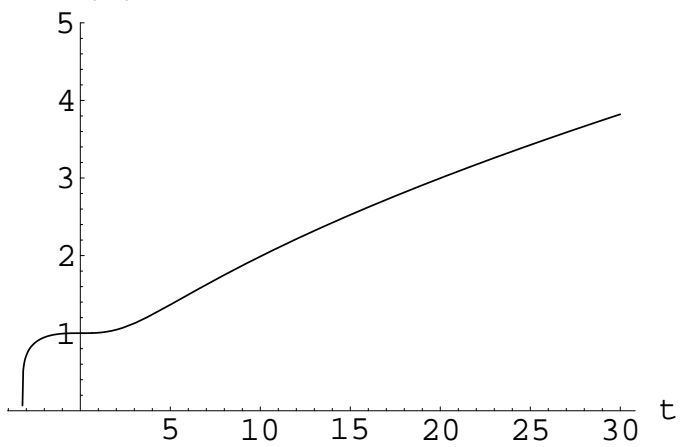

Figure 1: The expected value of the scale factor for Radiation, Dust, Cosmic strings and Domain walls dominated Universes (up left to down right respectively) in $a_{0}$ unit and $\gamma=1$.

with an equation of state $p_{\phi}=\rho_{\phi}$ near the signature changing hypersurface. Now, if the signature changing hypersurface is located at $t=0$, then in the vicinity of this point we have $\alpha_{\phi} \rightarrow 1$, or in the other words

$$
\lim _{\phi \rightarrow 0} U(\phi)=0
$$

On the other hand, to the reason of change of sign of the pressure and the energy density of the scalar field from Lorentzian to Euclidian region, the potential term in the neighborhood of signature changing hypersurface must be an odd function of $t$. An example of such kind of self interacting potential, which is introduced by Dereli and Tucker [12, is

$$
U(\phi)=\Lambda+a \sinh ^{2}(c \phi)+b \sinh (2 c \phi),
$$

where $a, b$ and $c$ are constant parameter. The first two terms in $U(\phi)$ give rise to a Sinh-Gordon scalar interaction. The third term breaks the symmetry of the potential under $\phi \rightarrow-\phi$, and is directly responsible for the signature changing properties of the solutions. 


\section{Conclusions}

In this work we have investigated a minisuperspace perfect fluid FRW quantum cosmological model in the context of signature change type spacetime. The use of Schutz's formalism for perfect fluid allowed us to obtain a SWD equation in which the only remaining matter degree of freedom plays the role of time. We found the eigenfunctions with arbitrary choices of factor ordering. Physically acceptable wave packets were constructed by appropriate linear combination of these eigenfunctions. The time evolution of the expectation value of the scale factor has been determined in the spirit of the many worlds and ontological interpretations of quantum cosmology. We have also explored the possibility of having solutions that are described by degenerate metrics signifying transition from a Euclidean to a Lorentzian domain at quantum level. Moreover, we have shown that adding a repulsive stiff matter to the classical scenario can reproduce the quantum signature changing results. Finally, we discussed the construction of the self interacting scalar fields which give rise to classical signature change scenario.

\section{References}

[1] J. B. Hartle, and S. W. Hawking, Phys. Rev. D 28, 2960 (1983).

[2] S. W. Hawking, Nuclear Physics B 239, 257 (1984).

[3] J. J. Halliwell, and J. B. Hartle, Phys. Rev. D 41, 1815 (1990).

[4] G. W. Gibbons, and J. B. Hartle, Phys. Rev. D 42, 2458 (1990).

[5] T. Dray, Int. J. Mod. Phys. D 6, 717 (1997).

[6] D. Hartley, R. W. Tucker, P. A. Tuckey and T. Dray, Gen. Rel. Grav. 32, 491 (2000).

[7] S. J. Campbell, J. Wainwright, Gen. Rel. Grav. 8, 987 (1977).

[8] G. Ellis , A. Sumeruk, D. Coule and C. Hellaby Class. Quantum Grav. 9, 1535 (1992).

[9] G. Ellis, Gen. Rel. Grav. 24, 1047 (1992).

[10] S. A. Hayward, Class. Quantum Grav. 9, 1851 (1992). 
[11] J. Martin, Phys. Rev. D 52, 6708 (1995), arXiv:gr-qc/9509053.

[12] T. Dereli and R. W. Tucker, Class. Quantum Grav. 10, 365 (1993).

[13] K. Ghafoori-Tabrizi, S. S. Gousheh and H. R. Sepangi, Int. J. Mod. Phys. A 15, 1521 (2000), arXiv:gr-qc/9910020.

[14] R. Mansouri, and K. Nozari, Gen. Rel. Grav. 32, 253 (2000).

[15] T. Dray G. Ellis, and C. Hellaby, Gen. Rel. Grav. 33, 1041 (2001).

[16] T. Dereli, M. Onder and R. W. Tucker, Class. Quantum Grav. 10, 1425 (1993).

[17] S. Jalalzadeh, F. Ahmadi and H. R. Sepangi, JHEP 08, 012 (2003), arXiv:hep-th/0308067.

[18] B. Vakili, H. R. Sepangi, JCAP 0509, 008 (2005).

[19] B. Vakili, S. Jalalzadeh, H. R. Sepangi, JCAP 0505, 006 (2005).

[20] F. Embacher, Phys. Rev. D 52, 2150 (1995), arXiv:gr-qc/9410012.

[21] F. Darabi and H. R. Sepangi, Class. Quantum Grav. 16, 1565 (1999), arXiv:gr-qc/9905008.

[22] M. Mars, J. M. M. Senovilla, and R. Vera, Phys. Rev. Lett. 86, 004219 (2001); Phys. Rev. D 76, 044029 (2007); Phys. Rev. D 77, 027501 (2008).

[23] P. Pedram and S. Jalalzadeh, Phys. Lett. B 659, 6 (2008), arXiv:0711.1996; P. Pedram, S. Jalalzadeh and S. S. Gousheh, Int. J. Theor. Phys. 46, 3201 (2007), arXiv:0705.3587; Phys. Lett. B. 655, 91 (2007), arXiv:0708.4143, Class. Quantum Grav. 24, 5515 (2007), arXiv:0709.1620; P. Pedram, M. Mirzaei, S. Jalalzadeh, and S. S. Gousheh, Gen. Rel. Grav., in press, arXiv:0711.3833.

[24] M. J. Gotay and J. Demaret, Phys. Rev. D 28, 2402 (1983); F. G. Alvarenga and N. A. Lemos, Gen. Rel. Grav. 30, 681 (1998); J. Acacio de Barros, N. Pinto-Neto, and M. A. Sagioro-Leal, Phys. Lett. A 241, 229 (1998); F. G. Alvarenga and N. A. Lemos, Gen. Rel. Grav. 31, 1743 (1999); F. G. Alvarenga, J. C. Fabris, N. A. Lemos, and G. A. Monerat, Gen. Rel. Grav. 34, 651 (2002).

[25] B. F. Schutz, Phys. Rev. D 2, 2762 (1970); Phys. Rev. D 4, 3559 (1971). 
[26] R. Arnowitt, S. Deser and C. W. Misner, Gravitation: An Introduction to Current Research, edited by L. Witten, Wiley, New York (1962).

[27] M. Kossowski and M. Kriele M, Class. Quantum Grav. 10, 1157 (1993).

[28] Dereli T and Tucker R W, Class. Quantum Grav. 10, 365 (1993).

[29] M. Kossowski and M. Kriele, Proc. R. Soc. Lond. Ser. A 444, 297 (1994).

[30] M. Kossowski and M. Kriele, Class. Quantum Grav. 10, 2363 (1993).

[31] V. G. Lapchinskii and V. A. Rubakov, Theor. Math. Phys. 33, 1076 (1977).

[32] Yudell. L. Luke, Integrals Of Bessel Functions (McGraw-Hill, New York, 1962), p 313.

[33] H. Everett, III, Rev. Mod. Phys. 29, 454 (1957).

[34] P. R. Holland, The Quantum Theory of Motion: An Account of the de Broglie-Bohm Interpretation of Quantum Mechanics, Cambridge University Press, Cambridge (1993).

[35] N. Pinto-Neto, Procedings of the VIII Brazilian School of Cosmology and Gravitation II, Edited by M. Novello (1999).

[36] A. B. Batista, J. C. Fabris, S. V. B. Gonçalves, J. Tossa, Phys. Lett. A 283, 62 (2001); Phys. Rev. D 65, $063519(2002)$. 